\title{
Epidemiological aspects of bovine trypanosomosis in an endemic focus of eastern Zambia: The role of trypanosome strain variability in disease pattern
}

\author{
Authors: \\ Justin Masumu $u^{1,2,3}$ \\ G. Tshilenge $\mathrm{e}^{1,2}$ \\ V. $\mathrm{Mbao}^{4}$

\section{Affiliations:} \\ ${ }^{1}$ Southern African Centre \\ for Infectious Disease \\ Surveillance, Chuo Kiikuu, \\ Morogoro, Tanzania \\ ${ }^{2}$ Laboratoire Vétérinaire \\ Central, Kinshasa, \\ Democratic Republic of the \\ Congo
}

${ }^{3}$ Institut National de Recherche Biomédicale, Avenue de la Democratie, Kinshasa I/RDC

${ }^{4}$ Centre for Ticks and Tickborne Diseases, Lilongwe, Malawi

Correspondence to: Justin Masumu

Email:

jmasumu@hotmail.com

Postal address:

PO Box 3297, Chuo Kiikuu,

Morogoro, Tanzania

How to cite this abstract: Masumu, J., Tshilenge,

G. \& Mbao, V., 2012

'Epidemiological aspects of bovine trypanosomosis in an endemic focus of eastern Zambia: The role of trypanosome strain variability in disease pattern', Onderstepoort Journal of Veterinary Research 79(2), Art. \#468, 5 pages. http:// dx.doi.org/10.4102/ojvr. v79i2.468

\section{Note:}

Proceedings of the

Conference of the Southern African Centre for Infectious Disease Surveillance 'One Health' held at the National Institute for Communicable Diseases, Johannesburg, July 2011.

(C) 2012. The Authors. Licensee: AOSIS OpenJournals. This work is licensed under the Creative Commons Attribution License.
Bovine trypanosomosis displays various epidemiological aspects in various areas. In some instances the disease has a high prevalence in animals with high impact on production whereas in other cases the disease has a low impact on production despite a high level of infection in animals. In addition epidemiological changes are frequently observed in various areas and are related to many factors including the vectors, the host, the parasites, the environment as well as the livestock management. However the implication of these factors in these changes is not fully elucidated. In eastern Zambia, factors predicting the establishment of severe infection in cattle are all present. However trypanosomosis occurring in cattle in this area has a low impact on livestock production. Several studies on the characterisation of trypanosome strains circulating in domestic and wild animals have been conducted in order to clarify the epidemiology of this disease in this area. These studies aimed at evaluating genetic and biological characteristics of these strains including their virulence profiles, their transmissibility by tsetse flies, their resistance to drugs and interference between different strains. In this review these findings are analysed in order to elucidate the implication of trypanosome strain variability in the distribution and the expression of this disease in the study area. The evolutionary trends of the situation occurring in this study area are also explained. Use of these findings is the context of disease control in the study area is further discussed.

\section{Bovine trypanosomosis in eastern Zambia: Background}

Bovine trypanosomosis is endemic in eastern province of Zambia. Previous epidemiological studies using parasitological, serological or molecular techniques showed that the prevalence of the disease varies in different districts (Machila, Sinyangwe \& Mubanga 2001; Sinyangwe, Delespaux \& Brandt 2004; Simukoko, Marcotty \& Phiri 2007). The same variation is observed within a particular district where a high prevalence of the disease is observed in some villages compared to other. In some cases no infection has been detected in cattle kept in some villages. In 1996, trypanosome infections were found in cattle in only $74 \%$ of sampling sites with a prevalence of infection varying between villages from $0 \%$ to $64 \%$ (Sinyangwe et al. 2004). Such variations are related to many factors including the habitat fragmentation of tsetse flies that affects tsetse density in various areas (Ducheyne, Mweempwa \& De Pus 2009). For example the decrease prevalence of the infection in cattle from 1998 to 2003 in Petauke where tsetse habitat has widely been fragmented supports this observation.

The temporal trends of the infection in cattle revealed no (Sinyangwe et al. 2004) or little (Simukoko et al. 2007) variations between seasons although higher prevalence seems to be observed during the rainy season (Simukoko et al. 2007) when tsetse flies are more abundant (Van den Bossche \& De Deken 2002). Such variations influence drug management and, although farmers treat their animals throughout the year, most drugs are selectively administered during this period (Van den Bossche, Doran \& Connor 2000).

In this area, the same selection approach is used by farmers in administrating trypanocides drugs in animals. In most cases oxen and cows are more treated (Van den Bossche et al. 2000) since these animals categories, especially oxen are more infected with trypanosomes (Simukoko et al. 2007). Although this selective treatment resulted in the reduction (1.5 treatments per year) of drugs administered to animals (Van den Bossche et al. 2000) it could not limit the development and spread of drug resistance in the area. From 1996 (Sinyangwe et al. 2004) to 2003 (Delespaux, Dinka \& Masumu 2008), drug resistance to diminazene aceturate increased fivefold although drug use didn't change during this period of time (Van den Bossche et al. 2000). Despite a high proportion of resistant strains in the area farmers continue using trypanocides which effect needs to be evaluated. 
Trypanosoma congolense remains the most prevalent trypanosome species affecting cattle in this area with more than $95 \%$ of the infections (Sinyangwe et al. 2004; Simukoko et al. 2007). In eastern province only the Savannah type has been previously characterised in livestock (Masumu, Marcotty \& Geysen 2006a). This type was previously shown to be highly virulent in cattle (Bengaly, Sidibe \& Ganaba 2002). Furthermore it is efficiently transmitted by Glossina morsitans morsitans, the main vector of trypanosomes in this area. Bloodmeal analyses revealed that cattle provide $75 \%$ of meals to G. m. morsitans in this area (Van den Bossche \& Staak 1997) and thus are highly exposed to infections with these highly virulent $T$. congolense Savannah strains circulating in this area. However the cattle breed (Angoni) reared on the plateau of eastern province are not trypanotolerant. Taken together, these factors, including a high level of resistance to trypanocides in trypanosome strains circulating in cattle and the restricted number of treatment per year, favour for the development of an epidemic situation in cattle on the plateau of eastern province. However economical surveys revealed that the disease has a low impact on livestock production (Doran 2000).

The isolation and characterisation of trypanosome isolates from cattle in this endemic area revealed sound features that explain the epidemiology of bovine trypanosomosis in this particular area. In this review trypanosome characteristics observed in this area will be analysed for their implication in the epidemiology of bovine trypanosomosis on the plateau of eastern province in Zambia.

\section{Biological characteristics of trypanosome strains circulating in eastern province}

Trypanosomes can only influence the distribution and expression of the disease in various areas and different hosts only if they are genetically and phenotypically different. In order to test this, T. congolense circulating in the domestic and wild animals in eastern province were characterised at genetic level (Masumu, Geysen \& Vansnick 2006b; Masumu, Geysen \& Van den Bossche 2009a) as well as for biological characters including the virulence profile (Masumu et al. 2006a; Van den Bossche, Chitanga \& Masumu 2011), the transmissibility by tsetse flies (Masumu, Marcotty \& Ndeledje 2006c; Masumu, Akoda \& Van den Bossche 2010) and the possible existence of cross-protection between different strains (Masumu et al., 2009). It appears that many strains of T. congolense circulate in the eastern province (Masumu et al. 2006b; 2009a). Very few isolates co-circulate in different areas indicating a more restricted distribution. In trypanosomes circulating in domestic animals most of the strains were of low virulence (Masumu et al. 2006a). Only 20\% of them were highly virulent. On the other hand most of the trypanosomes circulating in wild animals were highly virulent (Van den Bossche et al. 2011) highlighting the trypanotolerant trait of these animals. The presence of high prevalence of virulent strains in wild animals is also a clear indication of these animals being reservoirs of these strains in eastern province. In domestic cycle where trypanosomes circulate only in domestic animals, geographical distribution of these strains indicated that high virulent trypanosome strains are present in limited villages (4 out of 11) and different virulent profiles were observed in various areas suggesting an uneven distribution of those virulent trypanosome strains (Masumu et al. 2006a). Whether the circulation of low virulent trypanosome strains in domestic animals could protect them from the adverse affect of the high virulent strains was also assessed (Masumu et al. 2009b). Indeed there is a clear indication of the existence of cross-protection between trypanosomes of high and low virulence but the degree of protection varies in different combinations of strains. This finding suggests that the effect of virulent trypanosome strains may vary depending on the combination of the high and low virulent strains in a particular area. Only in areas where most virulent strains do not cross-react with the majority of low virulent strains will the disease have high impact on animal health and thus livestock production. In areas where high level of cross-protection is observed between strains of high and low virulence the effects of such virulent strains will be minimal. The epidemiological importance of the virulent strains was further stress by the fact they were efficiently transmitted by tsetse flies compared to the strains of low virulence (Masumu et al. 2006c).

\section{Involvement of the trypanosome strain variability in the geographic distribution of bovine trypanosomosis in eastern province}

The geographical distribution of trypanosomosis depend on multiple factors including the abundance and infection rate of tsetse flies, the livestock management, the level of drug resistance and drug use in a particular area and the degree of livestock to resist to tsetse fly bites as well as to trypanosome strains circulating in the area. In the context of eastern province, significant variations are observed in the distribution of tsetse in various areas. In most cases high prevalence of the disease is observed in areas highly infested by tsetse flies. Within the same geographic area such variations are related to the abundance of tsetse flies throughout the year. In eastern province it appears that tsetse flies are more abundant during the rainy season (Van den Bossche \& De Deken, 2002) explaining the high prevalence of the disease previously observed during this period (Simukoko et al. 2007). Such variations may also be explained by the variations observed in the strains of T. congolense circulating in these areas with a high prevalence of the disease likely being observed in areas where most trypanosome strains are efficiently transmitted by tsetse flies. Although highly virulent strains are efficiently transmitted that low virulent ones, further analyses revealed that during the chronic phase of infection the transmission of low virulent strains increases (Masumu et al. 2010). This indicates that high and low virulent strains are all transmitted by tsetse flies in this area since most low virulent strains induced chronic infections in cattle. 
The distribution of the disease can also be influenced by the level of drug resistance to trypanocides in strains circulating in various areas. It can be assumed that high prevalence of infection will be observed in areas where the level of drug resistance is also high. This can partially be explained by the persistence of infection after treatment with trypanocides in animals infected with resistant strains. In addition although there is not evident correlation between resistance and transmission to tsetse flies (personal observation) the level of drug resistance in individual trypanosome strains may influence the infection rate in individual tsetse flies in case of persistence of drugs in tsetse after feeding on treated animals prior infection through an infected bloodmeal. In eastern province high prevalence was observed in areas where the level of drug resistance was also high (Sinyangwe et al. 2004).

\section{Involvement of the trypanosome strain variability in the expression of bovine trypanosomosis in eastern province}

The expression of trypanosomosis in various areas varies considerably (Van den Bossche 2001). In most cases severe infections are observed when animals are kept near game areas whereas a rather mild infection occurs in areas where wild animals are rather absent. In eastern province bovine trypanosomosis is rather mild on the plateau but when livestock are kept near the Luangwa valley where wild animals are abundant they suffer from severe infections. This was previously suggested to result from the presence of heterologous challenge in wild areas as a consequence of the circulation of diverse strains in these areas (Van den Bossche 2001). Our findings revealed that even in areas where wild animals are absent several trypanosome strains circulate in domestic animals suggesting that the number of trypanosome strains is not important for the expression of the disease in a given area (Masumu et al. 2009a). Instead from the findings on virulence and cross-protection obtained in eastern province it appears that the expression of the disease in various areas is largely dependent on the virulence of trypanosome strains circulating in each particular area with severe disease being observed in areas where virulent strains are more prevalent. Our findings on high prevalence of virulent trypanosome strains in the wild environment compared to the plateau where wild animals are absent and where most strains are of low virulence support this observation (Van den Bossche 2001). However as indicated previously high level of interference between high and low virulent strains may attenuate the effects of the disease in animals previously infected with low virulent strains even after challenge with virulent strains (Masumu, Marcotty \& Geerts 2009b). The low impact the disease has on the plateau of eastern province may be explained by the circulation in animals of a high prevalence of low virulent trypanosome strains and the existence of a high level of cross-protection between the few virulent strains and the high prevalent low virulent strains. On the other hand the high prevalence of virulent strains in the wild environment explains the severity of the disease observed in these areas.

\section{Trypanosome strain variability and the development of drug resistance in eastern province}

The rapid development of drug resistance in eastern province arise many questions. On the plateau of eastern province a fivefold increase of drug resistance was observed from 1996 (Sinyangwe et al. 2004) to 2003 (Delespaux et al. 2008). In the absence of factors underlying the development of drug resistance in the area (Van den Bossche et al. 2000), such increase may be explained by the circulation of resistant strains between different areas. However our findings on genetic characterisation on the plateau indicate that the circulation of trypanosome strains is very limited (Masumu et al. 2009a). Even in geographically close villages similar strains were rarely observed. Indeed the circulation of trypanosome strains in various areas can be facilitated by the movement of the infected host or infected vectors. In this area livestock movement is very restricted. In addition tsetse flies do not move freely in various areas due to the fragmentation of their habitats. Variations in the distribution of tsetse flies are more linked to the distribution of cattle (Van den Bossche \& Staak 1997). These two factors are in favour of a more restriction of trypanosome strains circulating in various areas. Such restriction cannot facilitate the circulation of resistant trypanosome strains. Instead the development of drug resistance seems to be induced locally. Although genetic exchange was only reported in Trypanosoma brucei (Jenni, Marti \& Schweizer 1986) not T. congolense, it may be assumed that the spread of drug resistance in eastern province is related to genetic exchange (Delespaux, Dinka \& Masumu 2008) between resistant and susceptible trypanosome strains. Indeed resistant and susceptible trypanosome strains do circulate in animals belonging to the same village (Masumu et al. 2006a). Since two different strains can infect an individual animal, and tsetse flies can be infected more than once, the presence of two different strains in the same fly will favour the exchange of genetic material. Further studies are needed to clarify the role of genetic exchange in the spread of drug resistance.

\section{From the past to the future}

The high prevalence of low virulent trypanosome strains and the presence of high level of interference between strains of high and low virulence in eastern province, an area where tsetse flies take their blood meal principally from a susceptible animal i.e. cattle (Van den Bossche \& Staak 1997), is a perfect illustration of an endemic situation where the vector (tsetse flies), the parasite (trypanosomes) and the host (cattle) live in harmony. The current situation results from the fragmentation of tsetse habitat and the elimination of wild animals. Indeed many decades from now wild animals were prevalent in this area. Consequently the virulence profile of trypanosome strains was similar to that occurring in Luangwa valley where the trypanotolerant trait of wild animals selects against trypanosomes of low virulence. However the elimination of wild animals on this plateau 
led to a situation where tsetse depends on livestock for their survival (Van den Bossche \& Staak 1997) and trypanosome circulate only in susceptible animals. Since the presence of virulent strains induces severe infections in these susceptible animals, the later are either treated or die from their infection. This results in the elimination of these virulent strains and the subsequently high prevalence of strains that affect animal health to a lesser degree. Only virulent strains that can interfere with strains of low virulence can persist in the susceptible host animal thus explaining the persistence of $20 \%$ of the virulent strains in this area (Masumu et al. 2006a).

The situation prevailing on the plateau of eastern province indicates clearly that bovine trypanosomosis is endemic in this area. Although the prevalence of the disease varies largely in different areas (Machila et al. 2001; Sinyangwe et al. 2004; Simukoko et al. 2007), further changes are still to occur in the future in various areas. For example, Petauke district that had a high prevalence of the disease and a high prevalence of drug resistance in 1990s (Sinyangwe et al. 2004) is no longer the major focus of bovine trypanosomosis in eastern province. The high level of fragmentation resulting from the increased surface of cultivation of crops induced a spectacular reduction of tsetse fly abundance and subsequently a low prevalence in animals (Simukoko et al. 2007). On the other hand Katete district remains an area of concern since the level of infection in animals is still high (Simukoko et al. 2007) and more important the increase of drug resistance is alarming (Delespaux et al. 2008).

It's obvious that this situation is far from being stable in the near future. Indeed the presence of a wild environment in Luangwa valley where about $14 \%$ of wild animals are infected with trypanosomes (Anderson, Mubanga \& Fevre 2011) constitutes a serious threat to livestock production in eastern province. Although habitat fragmentation renders difficult to tsetse flies to move towards districts like Katete, Petauke, other district like Msoro that is located near the game is constantly at high risk of trypanosomosis. In addition this district may constitute a buffer zone between the high risk area of the valley and the plateau of eastern province. In this district the high challenge of tsetse flies and the tranfer of highly virulent trypanosome strains from the wild environment will result in a high infection rate in livestock including pigs and small ruminants that are rather resistant to trypanosomes. This was demonstrated in a survey where all livestock species (cattle, pigs, sheep and goats) kept in Msoro district were highly infected (> 10\%) with trypanosomes contrarily to the situation occurring on the plateau where most infections are restricted to cattle (Simukoko et al. 2007). Indeed in the absence of wild animals, the presence of such infections in livestock other than cattle is epidemiologically very important. First, in such areas of high risk of trypanosomosis farmers prefer exploiting small ruminants and pigs for their relative tolerance to T. congolense. Their relative number will exceed that of cattle. Further since these animals are trypanotolerant and rarely treated with trypanocides they will maintain trypanosomial infection for a long period of time and thus increase the risk of tsetse flies being infected with trypanosomes even virulent to cattle. Such risk will increase the severity of the disease in cattle in those particular areas explaining the habitual low production observed in cattle in such areas. Second this encroachment of people and livestock to the game area constitutes a new epidemiological situation where virulent trypanosome strains circulating in wild animals (Van den Bossche et al. 2011) will gradually be introduced into the livestock management area and finally reach the plateau of eastern Zambia. Whilst such movement of virulent trypanosome strains from the wild to the interface will be favoured by tsetse flies movement, the transfer of these strains from the interface to the plateau of eastern province will probably be occasioned by livestock movement. Since cattle movement is very restricted small ruminants and pigs will be the major factor for the circulation of trypanosomes between areas and the introduction of virulent trypanosome strains in various areas including the plateau where the situation is currently more endemic and rather stable. This will be facilitated for example by the commercial and social exchanges between families in the area that increases the movement of animals from one area to another. When infected with trypanosomes, pigs and small ruminants will favour the circulation of these virulent strains even in areas further away from the game areas. It would be advisable to conduct a follow up of trypanosome strains circulating on the plateau e.i. in Katete district where trypanosomes are more resistant but rather low virulent and at the interface e.i. in Msoro district where a high prevalence of virulent strains may be present whilst being less or no resistant to trypanocides.

\section{Trypanosome-related factors and disease control strategies}

Several control strategies have been used in eastern province for bovine trypanosomosis. The extent of drug resistance to either diminazene aceturate or isometamidium chloride indicates clearly that caution should be made in using these drugs to control trypansomosis in cattle. It is not well known how cattle withstand the infection in this particular area where drug use is minimal (Van den Bossche et al. 2000) and a high proportion of trypanosome strains are resistant to either or both drugs (Delespaux et al. 2008; Sinyangwe et al. 2004). Amongst the reasons is the circulation of a high prevalence of low virulent strains in livestock. Indeed infections induced by these strains have minimal effect on livestock production. In most cases low virulent strain can affect reproductive parameters but do not necessarily cause animal death. Like in theileriosis the presence of such infection can be beneficial in protecting animals against adverse effects of virulent strains.

In this area, farmers use production-oriented strategy where only sick animals are treated. Although this strategy could not reduce the development of drug resistance it reduces animal mortality (Van den Bossche et al. 2000). Our findings and the presence of high prevalence of resistant strains are in 
favour of such control strategy. Not only treating sick animals also is economically acceptable by resource-limited farmers, it favours the establishment of premonition state in animals that are subsequently protected against adverse effects of virulent strains. It's possible that this strategy will remain the strategic option used by farmers in the future although it does not boost the reproduction of infected animals (Van den Bossche et al. 2000). Such control strategy cannot be applied in areas displaying a different epidemiological situation like in Msoro or the game areas of Luangwa valley where the trypanosome profiles are rather threatening for livestock.

\section{Conclusion}

Many factors can influence the epidemiology of bovine trypanosomosis in various areas. Very few studies have so far been deeply conducted for the understanding of the involvement of the parasite in the epidemiology of this disease. Indeed this can only be possible if sound information is yielded at genetic and biological levels. This implies the development of appropriate tools for genotypic and phenotypic characterisation. In the situation prevailing on the plateau of eastern province of Zambia where wild animals are not present and tsetse flies depends largely on a susceptible host, the outcome of the disease on livestock health and production was found to be correlated with the profiles of trypanosome strains circulating in animals. Disease control through parasite management leading to the establishment of premonition state in animals is thus encouraged and further supports the control strategy so far adopted by farmers. Similar features can likely be observed in areas displaying a similar epidemiological situation although cautious is needed to generalise these findings. Finally the approach adopted here to clarify the epidemiology of a particular disease in a specific epidemiological condition can be applied to any disease provided appropriate tools are available for such studies.

\section{Acknowledgements Competing Interest}

The authors declare that they have no financial or personal relationship(s) which may have inappropriately influenced them in writing this paper.

\section{References}

Anderson, N.E., Mubanga, J., Fevre, E.M., Picozzi, K., Eisler, M.C., Thomas, R. et al. 2011, 'Characterisation of the wildlife reservoir community for human and anima trypanosomosis in the Luangwa Valley, Zambia', PLoS Negl Trop Dis 5, e1211. $\mathrm{http}: / /$ dx.doi.org/10.1371/journal.pntd.0001211
Bengaly, Z., Sidibe, I., Ganaba, R., Desquesnes, M., Boly, H. \& Sawadogo, L., 2002, 'Comparative pathogenicity of three genetically distinct types of Trypanosoma congolense in cattle: Clinical observations and haematological changes', Veterinary congolense in cattle: Clinical observations and haematological changes', Veterina
Parasitology 108, 1-19. http://dx.doi.org/10.1016/S0304-4017(02)00164-4

Delespaux, V., Dinka, H., Masumu, J., Van den Bossche, P. \& Geerts, S., 2008, 'Five-fold increase in Trypanosoma congolense isolates resistant to diminazene aceturate over a seven-year period in Eastern Zambia', Drug Resistance Updates 11 , 205-209. http://dx.doi.org/10.1016/j.drup.2008.10.002

Doran, M., 2000, 'Socio-economics of trypanosomosis', RTTCP, publishing, Bovine Trypanosomosis in Southern Africa, Harare 3, 156.

Ducheyne, E., Mweempwa, C., De Pus, C., Vernieuwe, H., De Deken, R., Hendrickx, G. et al., 2009, 'The impact of habitat fragmentation on tsetse abundance on the plateau of eastern Zambia', Preventive Veterinary Medecine 91, 11-18. http:// dx.doi.org/10.1016/j.prevetmed.2009.05.009

Jenni, L., Marti, S., Schweizer, J., Betschart, B., Lepage, R.W.F., Wells, J.M. et al., 1986 'Hybrid formation between African trypanosomes during cyclical transmission', Nature 322, 173-175. http://dx.doi.org/10.1038/322173a0

Machila, N., Sinyangwe, L., Mubanga, J., Hopkins, J.S., Robinson, T. \& Eisler, M.C., 2001, 'Antibody-ELISA seroprevalence of bovine trypanosomosis in the Eastern Province of Zambia', Preventive Veterinary Medicine 49, 249-257. http://dx.doi. org/10.1016/S0167-5877(01)00178-7

Masumu, J., Akoda, G. \& Van den Bossche, P., 2010, 'Transmissibility, by Glossina morsitans morsitans, of Trypanosoma congolense strains during the acute and chronic phases of infection', Acta Tropica 113, 195-198. http://dx.doi. org/10.1016/j.actatropica.2009.09.002

Masumu, J., Geysen, D. \& Van den Bossche, P., 2009a, 'Endemic type of anima trypanosomosis is not associated with lower genotypic variability of Trypanosoma congolense isolates circulating in livestock', Research in Veterinary Science 87, 265-269. http://dx.doi.org/10.1016/j.rvsc.2009.03.003

Masumu, J., Marcotty, T., Geerts, S., Vercruysse, J. \& Van den Bossche, P., 2009b, 'Cross-protection between Trypanosoma congolense strains of low and high virulence', Veterinary Parasitology 163, 127-131. http://dx.doi.org/10.1016/j. vetpar.2009.04.006

Masumu, J., Marcotty, T., Geysen, D., Geerts, S., Vercruysse, J., Dorny, P., et al., 2006a, 'Comparison of the virulence of Trypanosoma congolense strains isolated from cattle in a trypanosomosis endemic area of eastern Zambia', International Journa for Parasitology 36, 497-501. http://dx.doi.org/10.1016/j.ijpara.2006.01.003

Masumu, J., Geysen, D., Vansnick, E., Geerts, S. \& Van den Bossche, P., 2006b, 'A modified AFLP for Trypanosoma congolense isolate characterization', Journal of Biotechnology 125, 22-26. http://dx.doi.org/10.1016/j.jbiotec.2006.01.016

Masumu, J., Marcotty, T., Ndeledje, N., Kubi, C., Geerts, S., Vercruysse, J., Dorny, P. \& Van den Bossche, P., 2006c, 'Comparison of the transmissibility of Trypanosoma congolense strains, isolated in a trypanosomosis endemic area of eastern Zambia, by Glossina morsitans morsitans', Parasitology 133, 331-334. http://dx.doi. org/10.1017/S0031182006000369

Simukoko, H., Marcotty, T., Phiri, I., Geysen, D., Vercruysse, J. \& Van den Bossche, P., 2007, 'The comparative role of cattle, goats and pigs in the epidemiology of livestock trypanosomosis on the plateau of Eastern Zambia', Veterinary Parasitology 147, 231-238. http://dx.doi.org/10.1016/j.vetpar.2007.04.005

Sinyangwe, L., Delespaux, V., Brandt, J., Geerts, S., Mubanga, J., Machila, N. et al., 2004, 'Trypanocidal drug resistance in Eastern province of Zambia', Veterinary Parasitology 119, 125-135. http://dx.doi.org/10.1016/j.vetpar.2003.11.007

Van den Bossche, P., Chitanga, S., Masumu, J., Marcotty, T. \& Delespaux, V., 2011, 'Virulence in Trypanosoma congolense Savannah subgroup. A comparison between strains and transmission cycles', Parasite Immunology 33, 456-60. http://dx.doi.org/10.1111/j.1365-3024.2010.01277.x

Van den Bossche, P. \& De Deken, R., 2002, 'Seasonal variations in the distribution and abundance of the tsetse fly, Glossina morsitans morsitans in eastern Zambia' Medical and Veterinary Entomology 16, 170-176. http://dx.doi.org/10.1046/ j.1365-2915.2002.00360.x

Van den Bossche, P., 2001, 'Some general aspects of the distribution and epidemiology of bovine trypanosomosis in southern Africa', International Journal for Parasitology 31, 592-598. http://dx.doi.org/10.1016/S0020-7519(01)00146-1

Van den Bossche, P., Doran, M. \& Connor, R.J., 2000, 'An analysis of trypanocidal drug use in the Eastern Province of Zambia', Acta Tropica 75, 247-258. http://dx.doi. org/10.1016/S0001-706X(00)00059-0

Van den Bossche, P. \& Staak, C., 1997, 'The importance of cattle as a food source for Glossina morsitans morsitans Westwood (Diptera: Glossinidae) in Katete District, Eastern Province, Zambia', Acta Tropica 65, 105-109. http://dx.doi.org/10.1016/ S0001-706X(97)00658-X 